\title{
Novel association between FOXO3 rs2232365 polymorphism and late-onset preeclampsia: a case-control candidate genetic study
}

Xuefeng Pan ${ }^{1 \dagger}$, Benjie Wei ${ }^{2+}$, Hong Wang ${ }^{1}$, Lingyu Ma', Zhaoli Du² and Ying Chen ${ }^{1 *}$ (D)

\begin{abstract}
Background: Both genetic susceptibility and dysregulated lipid metabolism are important susceptibilities to preeclampsia. In the study, we devote to investigate the associations of FOXO3 and TLR7 genetic polymorphisms with preeclampsia in a Chinese population.

Methods: This case-control study involved 335 Han Chinese pregnant women, including 177 pregnant women with preeclampsia and 158 healthy controls. The preeclampsia group was further sub-grouped into early-onset preeclampsia (EOPE, $n=70$ )and late-onset preeclampsia ( $L O P E, n=107$. Three single nucleotide polymorphisms (SNPs), including FOXO3 (rs2232365, rs3761548), and TLR7 rs3853839 were genotyped by multiplex PCR for targeted next-generation sequencing. The $x^{2}$ test and multiple interaction effect analyses were performed to determine the association of three SNPs with serum lipid levels and thyroid function in women with preeclampsia.

Results: The genotype (CC vs. $T+C T)$ distribution of rs2232365 revealed a significant association with LOPE $(P=$ 0.004 , odds ratio $=3.525(0.95 \mathrm{Cl}: 1.498-8.164))$. No significant difference was found in the genotype and allele frequencies of rs3761548 and rs3853839 between controls and cases ( $P>0.05)$. Moreover, the genotype CT/TT of rs2232365 was significantly correlated with increased TG/HDL levels in the LOPE group ( $p=0.014)$.

Conclusions: The polymorphisms of rs2232365 are associated with the risk of LOPE and may modulate TG/HDL levels in pregnant women with LOPE.
\end{abstract}

Keywords: Preeclampsia, Single nucleotide polymorphism, rs2232365, BMI, Lipid metabolism

\section{Introduction}

Preeclampsia (PE) is a pregnancy-specific syndrome and associated with significant maternal and fetal morbidity and mortality [1]. PE averagely affects $6.7 \%$ of pregnant women globally and $4.2 \%$ individuals in China [2, 3]. The pathogenesis of $\mathrm{PE}$ is multifactorial, with acknowledged

\footnotetext{
* Correspondence: yingchen@jlu.edu.cn

${ }^{+}$Xuefeng Pan and Benjie Wei contributed equally to this work.

'Department of Obstetrics, The First Hospital of Jilin University, Xinmin Street 1, Changchun, Jilin Province 130021, China

Full list of author information is available at the end of the article
}

contributions by genetic susceptibility, inflammatory stimuli, metabolic syndrome, oxidative stress, placental, and vascular dysfunction [4]. According to epidemiological studies, there was a high prevalence of metabolic syndrome in women with PE [5], otherwise autoimmune diseases and dysregulated lipid metabolism showed a tight association [6]. One of these most important hypotheses of $\mathrm{PE}$ is that maternal adequate immunological response is necessary to the existence of the fetus in pregnancy [7].

Forkhead/winged helix transcription factor(FoxP3)is the key transcription factor for Regulatory T cells (Tregs)

C C The Author(s). 2020 Open Access This article is licensed under a Creative Commons Attribution 4.0 International License, which permits use, sharing, adaptation, distribution and reproduction in any medium or format, as long as you give appropriate credit to the original author(s) and the source, provide a link to the Creative Commons licence, and indicate if changes were made. The images or other third party material in this article are included in the article's Creative Commons licence, unless indicated otherwise in a credit line to the material. If material is not included in the article's Creative Commons licence and your intended use is not permitted by statutory regulation or exceeds the permitted use, you will need to obtain permission directly from the copyright holder. To view a copy of this licence, visit http://creativecommons.org/licenses/by/4.0/ The Creative Commons Public Domain Dedication waiver (http://creativecommons.org/publicdomain/zero/1.0/) applies to the data made available in this article, unless otherwise stated in a credit line to the data. 
differentiation and function [8]. Tregs, as a specialized subset of immune cells, plays an important role in the establishment and maintenance of immune tolerance [9]. FoxP3 is responsible for the differentiation of Tregs to a suppressive phenotype and stabilizing their lineage [10]. There are several conserved noncoding sequences, designated as CNS 0-3, in the genomic region of Foxp3 locus. CNS 0-3 holds different signaling pathways respectively and deficiency of FoxP3 will impair the suppressive activity of Tregs [11]. A meta-analysis study showed that forkhead box protein 3 polymorphisms (rs2232365, rs3761548) were associated with the outcome of immunerelated pregnancy complications. Immunological incompatibility between mother and fetus is frequently observed in preeclampsia and genetic factors related to the immunological pathway in preeclampsia have been discovered [12]. In Asian, rs3761548 polymorphism was significantly associated with multiple sclerosis, an immune-related central nervous disease [13]. In the Chinese Han population, rs2232365 and rs3761548 polymorphisms confer an important susceptibility to unexplained recurrent spontaneous abortion by altering Foxp3 function and/ or its expression [14].

Toll-like receptors(TLRs)is critical innate immune activators, which can affect Treg-dependent immune regulation by reducing the number of Tregs $[15,16]$. TLRs, as innate immunity sensors, play important roles in the activation of innate and adaptive immune responses [17]. Polymorphisms of TLR7 rs3853839 are associated with the susceptibility to Chikungunya virus (CHIKV) infection in Indian people and the severity of EV71 in Chinese boys $[18,19]$.

Although the precise mechanism of preeclampsia is not understood, the disease is thought to occur as soon as the placenta was implanted [20]. Delay in childbearing, obesity, metabolic disorders, and genetic risk are all involved in this development of preeclampsia [21]. This study aimed to investigate whether rs3853839, rs2232365, and rs3761548 located in the X-chromosome are associated with preeclampsia in a Chinese case-control cohort, with a specific focus on the biochemical metabolic parameters.

\section{Material and methods}

\section{Study subjects}

The study included 335 individuals who provided informed written consent, including 177 pregnant women with preeclampsia and 158 healthy controls. The patient group was further classified into two subsets: 70 patients with early-onset preeclampsia (EOPE, defined as preeclampsia diagnosed within $34+0$ weeks of gestation) and 107 patients with late-onset preeclampsia (LOPE, defined as preeclampsia diagnosed after $34+0$ weeks of gestation) following the latest ACOG Practice Bulletin No. 202: Gestational Hypertension and Preeclampsia
[22]. The diagnostic criteria for PE is defined as blood pressure $\geq 140 / 90 \mathrm{mmHg}$ after 20 weeks of gestation. Severe hypertension is defined as blood pressure $\geq 160$ / $110 \mathrm{mmHg}$ with or without proteinuria, accompanying the following symptoms: liver injury, renal insufficiency, pulmonary edema, cerebral or visual disturbance. This research project was performed in accordance with the Declaration of Helsinki, and ethical approval was obtained from the local Ethics Committee of the First Hospital of Jilin University, Changchun, China (Permission number: 2018 -401). All individuals were from the Han population in Northeast China and signed a written informed consent form. Figure 1 shows a chart of the trial design.

Exclusion criteria included patients with diabetes mellitus, chronic hypertension, diseases of the blood system, cardiovascular diseases, renal disease, and cerebrovascular accidents. Pregnant women exhibiting elevated blood pressure without proteinuria were also excluded from the study. All the controls were confirmed with normal blood pressure and without any other chronic diseases.

\section{Patient and public involvement}

This case-control study was conducted between May 2018 and January 2020 in the department of Obstetrics of the First Hospital of Jilin University in China. Using logistic regression, our study involved 15 explanatory variables, and since the minimum observations required are multiplied by 10 to yield significant results, our studied sample size was determined as above 300 cases. The design of this study was not directly involved in the diagnosis and treatment of patients, and the intervention is not considered to affect the patient's therapy. Patients in hospital were recruited voluntarily when they received medical history collection. The meaningful results for therapeutic effect were disseminated to study participants, such as lipid metabolism and thyroid function, and uncertain genetic information were reserved. In the study, no additional burden were added to those patients themselves for the funding supported the Jilin Province Science Development Plan.

\section{Measures}

Gestational age was calculated by the last menstrual period and the first ultrasound in the first trimester. Systolic blood pressure (SBP), diastolic blood pressure (DBP), height, and weight of each patient were measured before treatment according to the Seventh Report of the Joint National Committee on Prevention, Detection, Evaluation, and Treatment of High Blood Pressure [23]. Venous blood samples $(3 \mathrm{~mL})$ from every participant were obtained from the subjects after a 12-hour fast. The circulation levels of thyrotropin, thyroid-stimulating 


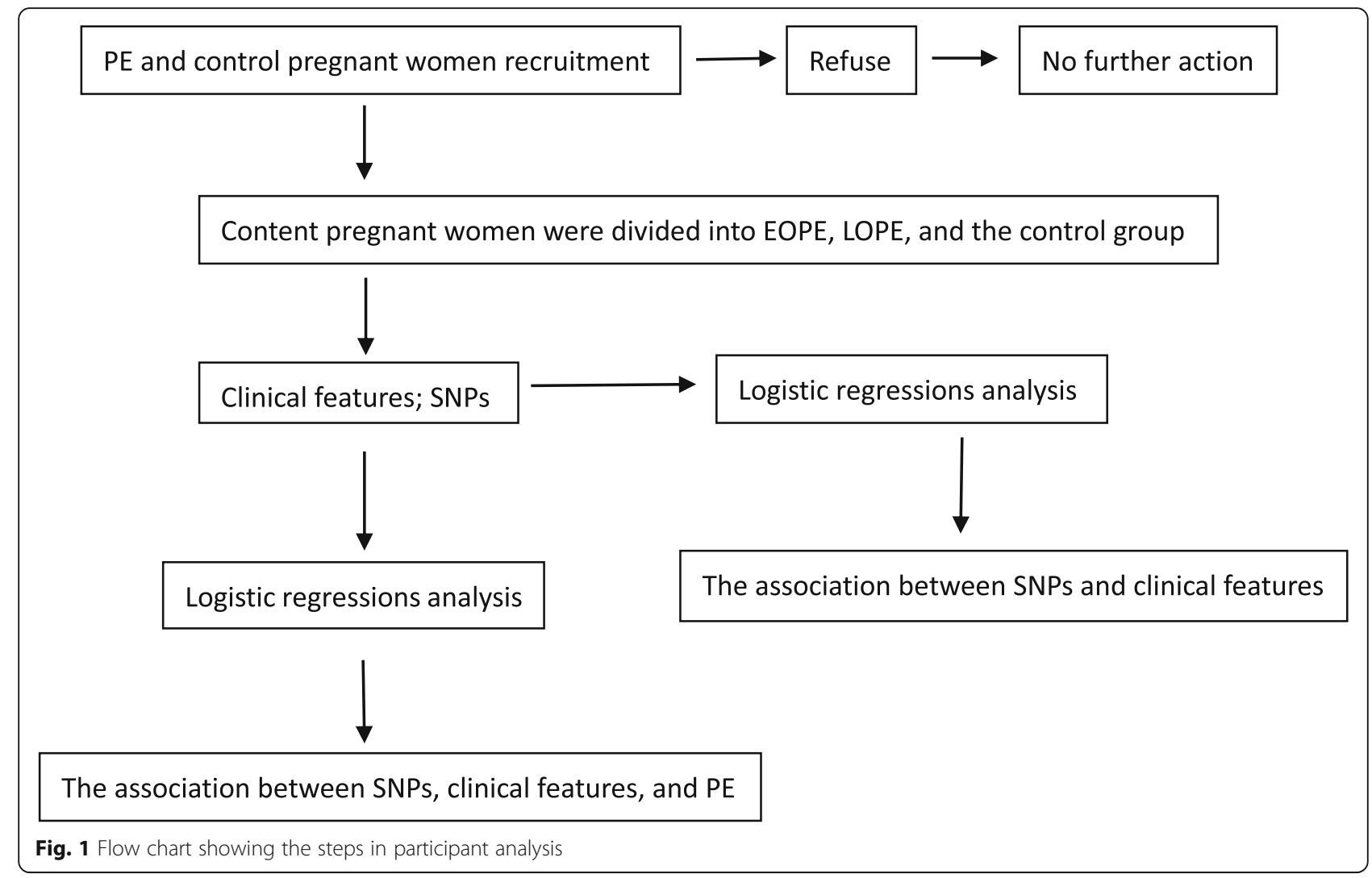

hormone(TSH), triiodothyronine (T3), thyroxine(T4), triglyceride(TG), total cholesterol(TC), high-density lipoprotein cholesterol (HDL-C), and low-density lipoprotein cholesterol (LDL-C) were measured by an automatic biochemical analyzer (SEKISUI medical technology Ltd., Tokyo, Japan).

\section{DNA extraction and genotyping}

Genomic DNA was extracted from $3 \mathrm{~mL}$ EDTCanticoagulated blood samples using a Gene JET Whole Blood Genomic DNA Purification kit (Thermo Scientific Co. Ltd.). SNPs were analyzed through multiplex PCR for targeted next-generation sequencing.

\section{Statistical analysis}

All statistical analyses were performed using Microsoft Excel (2007) and IBM SPSS Statistics for Windows (version 18.0, IBM Corporation., Armonk, NY, USA). Genotype numbers were determined by manually counting and allele frequency was determined from the genotype frequencies. The calculation for Hardy-Weinberg equilibrium was performed and genotype distribution was determined. (available at http://ihg.gsf.de/cgi-bin/hw/hwal.pl). Normality distributed continuous variables were presented as mean \pm standard deviation (SD) for parametric variables, and continuous variables with non-normal distribution are represented by the median (Q1-Q3). Non-normal distribution data were compared between the studied groups using U-test. The chi-square test was used to assess the associations of genotypes and alleles with preeclampsia. Haplotype analysis was predicted from genotype data by the computer program Haploview. Binary logistic regression analysis was also performed to identify independent risks of preeclampsia and linear regression analysis was used for the risk of high TG/ HDL in LOPE. The odds ratios (ORs) and 95\% confidence intervals (CIs) were calculated, and $P<0.05$ for all pairwise comparisons; $P<0.017$ for the multiple comparisons, according to Bonferroni correction were considered significant.

\section{Results}

\section{Clinical characteristics}

The general characteristics of all participants, including controls, EOPE, and LOPE, were presented in Table 1. None of them was missing and all blood samples were tested successfully. There were significant differences in multiple parameters between the PE group and the control group, but the differences were not consistent. Pregnant women with EOPE had higher levels of BMI, TSH, FT3 and TG/HDL than controls. In the LOPE subgroup, weight, BMI, TSH, FT3, TG, TG/HDL were different from those of 


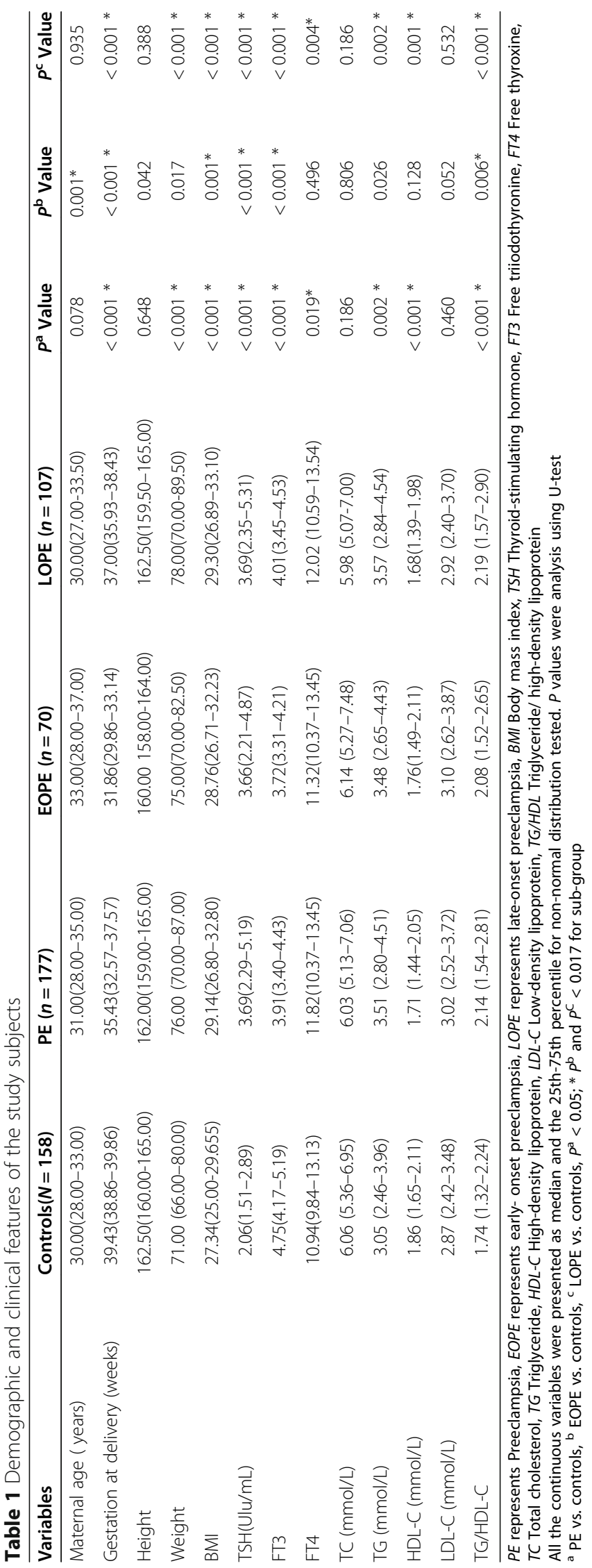


the control group. $P<0.05$ for all pairwise comparisons; $P<0.017$ for the multiple comparisons, according to Bonferroni correction.

\section{Genotype and allele frequencies of cases and controls} Genotype frequencies of cases and controls are shown in Table 2. Hardy-Weinberg equilibrium was tested in the control group, and the result was consistent with the expectation. There was no significant difference of other genotypic frequencies between women with PE or EOPE and controls. Although the variants of rs2232365 and rs3853839 showed no significant association with LOPE risk $(P>0.017)$, which was also brought into binary logistic regressions to analyze the association between LOPE and different parameters.

There was not a significant association between the $\mathrm{C}$ allele of rs2232365 and LOPE risk in Chinese Han pregnant women $(P=0.021)$, but binary logistic regressions still were calculated. Furthermore, similar associations were also observed in EOPE groups. We did not find any relationships between alleles of SNPs rs3853839 and rs3761548 and PE.

\section{Association between genotype variants and clinical and biochemical parameters}

Table 3 depicted the laboratory parameters according to the significant SNPs in LOPE individuals. LOPE patients with CC genotypes of rs2232365 showed significantly higher levels of HDL and lower TG/HDL compared to $\mathrm{TT}+\mathrm{CT}$ genotypes. Furthermore, significantly lower levels of T3 were found in the GG genotype of rs3853839 between LOPE patients as compared to the controls $(P=0.005)$, while the parameter showed no association in the analysis of linear regression. However, no significant differences were observed between laboratory factors and SNPs rs3761548 in LOPE subjects ( $P>0.05$ for all comparisons). In EOPE, no significant associations were observed between genotype and biochemical parameters, which was not shown here.

\section{Association between PE and different parameters}

Binary logistic regressions were performed and the results were presented in Table 4. These significant associations appeared after the false-positive discovery amendment. We observed that both SNP rs2232365 (CC) and TG/HDL were associated with LOPE. Linear regressions were further performed to analyze the association between TG/HDL and other parameters, and our study found that SNP rs2232365 related to it based on current data $(p=0.027,(0.95 \mathrm{CI}: 0.089-1.430))$ in those patients with LOPE. To assess exposure risk, extra risk were calculated by converting the odds ratio. The extra risk was 55\%, 63.8\% and 58.7\% higher in the PE, EOPE and LOPE groups, respectively, compared with the control group. We especially assess the absolute risks of CC genotype LOPE, those cases with CC genotype has increasing risk as high as $18 \%$ compared with those with CT/TT genotype. The extra risk of TG/HDL was $34.2 \%$ in LOPE group (showed in Fig. 2).

\section{Discussion}

\section{Summary of key results}

In this study, we tested the correlation of 3 specific Xchromosome-related SNPs with the susceptibility of PE and identified FOXP3 rs2232365 as a novel risk factor of LOPE. Pregnant women with TT/CT genotype had higher TG/DHL in LOPE, which was the first reported and disclosed implicit relationship(Tables 2 and 3 ). We observed the significant associations between LOPE and rs2232365 (CC), BMI, TSH, FT4, TG, and TG/HDL by multiple logistic regressions and TG/HDL ratio was higher in LOPE patients with CC genotype. These findings indicate that we can develop some personalized treatment plans for our patients who were high-risk ones screened by prediction models. For decreasing TG/HDL to reduce the incidence of late-onset preeclampsia, we can enhance diet and exercise management in those women with high-risk factors, especially in those cases with rs2232365 (TT/CT) genotype.

Although multiple studies have investigated mechanisms of $\mathrm{PE}$, and they remain unclear. The balance of immune plays an important role in pregnancy from placentation to delivery. Some papers on oocyte donation (OD) during in vitro fertilization (IVF) cycles show strong evidence that immunity may be involved in $\mathrm{PE}$ development. Increased risk of EOLP for pregnant women with oocyte donation (OD) has been observed compared with both spontaneous pregnancies and pregnancies achieved by in vitro fertilization (IVF) with autologous oocytes [24]. The decidua obtained by OD, as a kind of completely allogeneic but partially maternal, showed more genetic and immunological differences and need a more intense downregulation of the maternal alloimmune response [25]. Aberrant human lymphocyte antigen (HLA) allogenicity can alter the function of uterine natural killer cells (uNK) and lead to the abnormal maternal blood supply to the placenta, which is the predominant cause of facilitates disorders such as PE and fetal growth restriction [26]. A recent study also showed abnormally low uterine arteries pulsatility index and serum maternal $17 \beta-\mathrm{E}$ at 11 to $13+6$ weeks in oocyte donations as compared to natural conceptions [27, 28]. The authors of this study described this new phenomenon as increasing placental perfusion to compensate for maternal or placental dysfunction [27]. In animal models, the fluctuation of circulating steroids could lead to a reduction in uterine vascular resistance [29]. In addition, a recent study showed that the increased age of a pregnant woman 


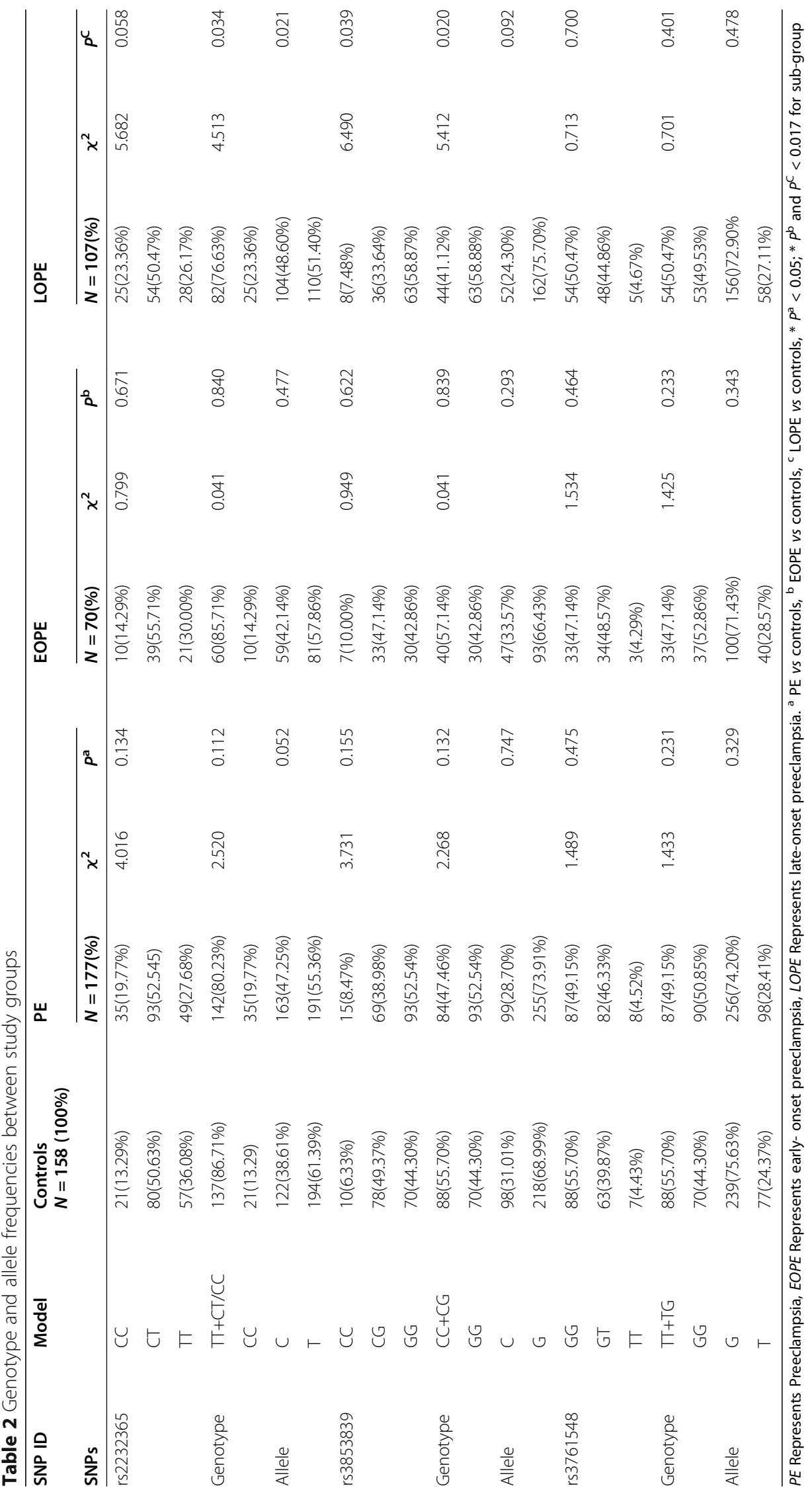




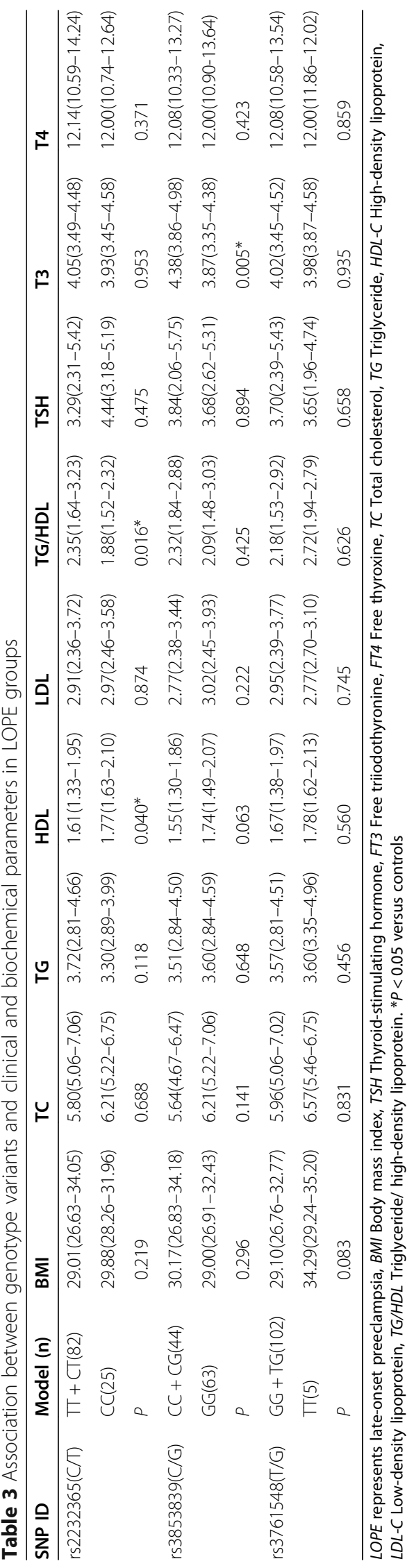


Table 4 Odds ratios (95\% confidence intervals) for the association between PE and different parameters

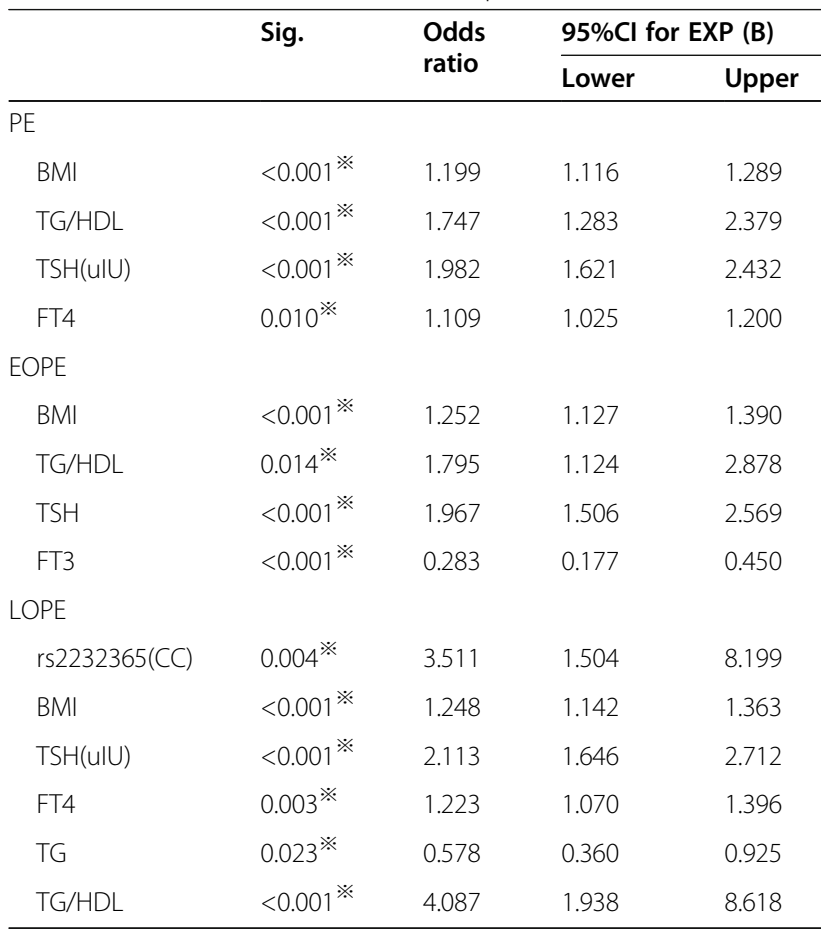

$P<0.05$ was considered statistically significant. PE represents preeclampsia, EOPE represents late-onset preeclampsia, LOPE represents late-onset preeclampsia, BMI Body mass index, TSH thyroid-stimulating hormone, $F T 3$ Free triiodothyronine, FT4 Free thyroxine, TG Triglyceride, HDL High-density lipoprotein, TG/HDL Triglyceride/ high-density lipoprotein

during egg donation increases the risk of PE [29]. The above discoveries seem to explain the hypothesis that older pregnant women whose lower $17 \beta-\mathrm{E}$ affect the uterine arteries pulsatility index had a higher risk for PE.
Maternal $\mathrm{T}$ lymphocytes play an important role in immune response and keep a transient state of tolerance for paternal alloantigens [30]. It has been demonstrated that Treg cell participates in maintaining homeostasis and preventing maternal immune self-reactivity during normal pregnancy [31]. Loss-of-function mutations of the FOXP3 gene can conduce to the functional deficiency of Treg cells in animal and human models [32], which can further inhibit natural killer cells, macrophages, and dendritic cells to affecting the maternal immune tolerance [33]. SNP rs2232365 located in a putative binding site for the transcription factor GATA-3 and its polymorphism was likely to contribute to variant(s) in the quantity or quality of FOXP3 [33]. FOXP3 gene and pregnancy have been extensively studied and proved to be associated with recurrent pregnancy loss in Egyptian [33] and preterm premature rupture in the Zaporizhzhia population [34]. A meta-analysis about the association between the SNP rs2232365 and immune-related pregnancy complications revealed that allele G and GG or AG genotype were highrisk factors for adverse pregnancy outcomes [35]. In the present study, we identified rs2232365 was associated with a higher risk of LOPE in Northeast women in China and affected the metabolism of lipids.

Immune status is generally correlated with heredity, BMI, lipid metabolism, and nutrition. The level of FOXP3 was markedly elevated in patients with PE who hold abnormal maternal lipids, hyperglycemia, and high BMI [36]. HDL is a vasodilator that interacted with the vascular endothelium and its concentration generally increases throughout the whole pregnancy [37]. HDL carries redundant potentially harmful cholesterol to the liver to excrete reverse cholesterol and protect the

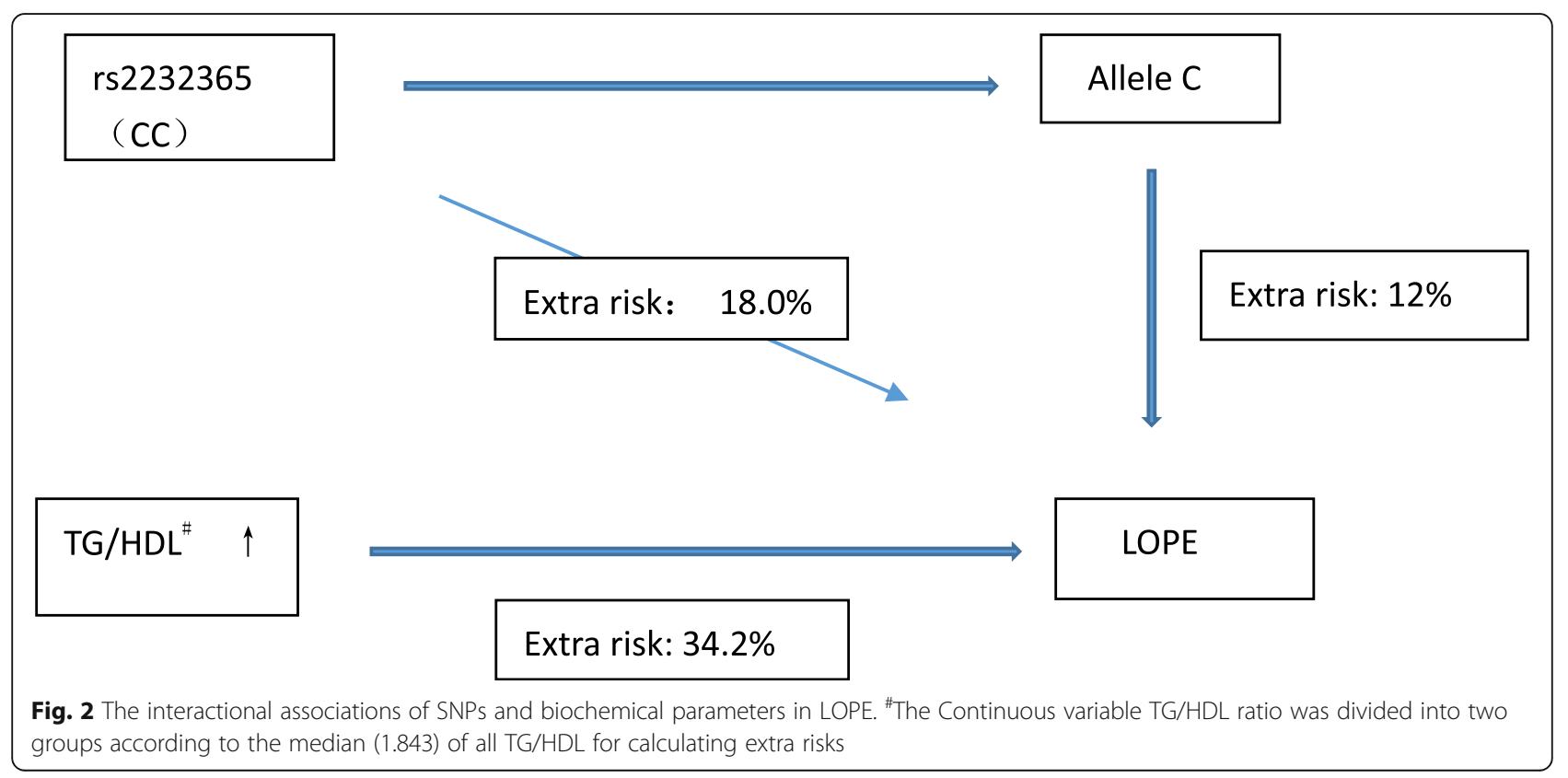


maternal vascular endothelium [38]. In the present study, significantly higher levels of serum TG were identified in patients with $\mathrm{PE}$, consistent with previous studies [36]. Therefore, TG/HDL as the risk factor for both EOPE and LOPE, can conveniently reflect the balance between dangerous and protective lipids in patients. We find FOXP3 rs2232365 a novel function of affecting the TG/HDL level in Chinese pregnant women.

FOXO3 rs3761548 was also reported as a risk factor to immune-related pregnancy complications [35] and an important contributor for the progression of PE in Iranian women [39]. While no associations between SNP rs3761548 and preeclampsia were found either in Iranian women [40] or the Turkish population [41]. We thought that the conflicting observations were conduced mainly by ethnic and geographic differences. Mutation of the genotype of rs3761548 mostly affects the expression and activity of FOXP3 protein, which was further involved in many autoimmune diseases including rheumatoid arthritis [42], allergic rhinitis [43], and autoimmune thyroid disease [44]. Our study showed that FOXO3 rs3761548 was not found to be related to preeclampsia in Northeast women of China based on the present date.

Another TLR7 rs3853839 significantly associated with LOPE was found by $x^{2}$ test, which was also related to the levels of FT3. But, the difference did not appear after a logistical regression analysis based on present data. Toll-like receptors (TLRs) which are a family of patternrecognition receptors promote the activation of autoreactive $B$ cell and elicit innate/adaptive immune responses [45]. Female patients with rs3853839 CC genotype might present a pronounced defensive effect against persistent HCV infection [46] and periodontitis [47]. Otherwise, allele $C$ and SNP rs3853839 are associated with severe hand, foot, and mouth disease (HFMD) [48]. In Chinese women, there was no association between rs3853839 and preeclampsia, and the relationship of FOXP3 gene rs3853839 and thyroid function need further research involving larger samples.

BMI, Thyroid dysfunctions, and dyslipidemia were enrolled in the control-study, and there were significant associations between those parameters and preeclampsia, including EOPE and LOPE subgroups. Pregnancy women who exposed to dyslipidemia are more prone to developing gestational diabetes, preeclampsia, preterm birth, or cardiovascular diseases (CVD) $[49,50]$. Thyroid dysfunction, including hypothyroidism and thyrotoxicosis, is associated with preeclampsia, preterm delivery, placental abruptions, and fetal neurologic development [51]. Pre-pregnancy BMI had been identified to be independent risk factors for both EOPE and LOPE, and BMI might be one of the ways to diagnose preeclampsia [52]. Our results are similar to those of previous studies.
Changes in thyroid function profiles in women with preeclampsia are controversial in different studies reported. The levels of T3 and T4 hormones are higher in Sudanese patients with preeclampsia [53], but not in Iranians [54]. In our study, we got a consistent and stable result: women with preeclampsia had higher levels of TSH and lower FT3 hormones. The levels of FT3 and FT4 are associated with the tendency of preeclampsia, although the information regarding thyroid function in preeclampsia was scanty [55].

Preeclampsia, known as a complex disease, involves multiple risk factors including genetic susceptibility, immunity, hypothyroidism, and environmental factors. Those predictive values of the traditional screening models of PE were population dependent, basing on maternal medical histories, characteristics, and biophysical and biochemical markers. The Fetal Medicine Foundation (FMF) algorithm had been identified to be super to the method of the National Institute for Health and Care (NICE), and the DRs (95\% CI) for EOPE and LOPE were $58.2 \%(45.5-70.2)$ and $44.1 \%(37.3-51.1)$ respectively [24] To date, no single risk factor as the absolute predictive indicator has been identified. We think that the onset of the disease is based on the cumulative contributions of many risk factors. Pregnant women with high-risk factors should be screened out relying on a predictive model of preeclampsia. According to the individual genetic background of patients, the regulation of their environmental risk maybe a personalized diagnosis and treatment measure worth further study. China is a multi-nationality county with different genetic information, and Chinese Northern Han populations were our targeted populations. In the present study, we identified different risk factors for EOPE and LOPE through the method of traditional logistic regression. Different risk factors are shown in the study, which means there are different pathogenesis in EOPE and LOPE. We think it is a considerable method to predict the risk of preeclampsia based on the cumulative effect of different risk factors. In the further, we will continue to explore the risk factor of preeclampsia to build a model of preeclampsia. Further studies for genetic polymorphism described in maternal-fetal pairs of spontaneous conceptions and oocyte donations will help us to better etiological understanding of PE.

The major limitation of the present study should be noted. That the sample size is relatively small, which will be expanded in both controls, and PE groups with more environmental and genetic factors for optimization and validation of the predictive model.

\section{Conclusions}

A novel function was found that pregnant women with TT/CT genotype of FOXO3 rs2232365 had 
higher TG/HDL in LOPE. C allele and CC genotype of SNP rs2232365 are associated with the risk of LOPE in Chinse northeast women.

\author{
Abbreviations \\ SNP: Single nucleotide polymorphism; FOXO3: Forkhead/winged helix \\ transcription factor; TLR7: Toll-like receptors; Tregs: Regulatory T cells; \\ PE: Preeclampsia; EOPE: Early-onset preeclapmsia; LOPE: Late-onset \\ preeclampsia; BMI: Body mass index ; HFMD: Mouth Disease; \\ CVD: Cardiovascular diseases; TSH: Thyroid-stimulating hormone; \\ T3: Triiodothyronine; T4: Thyroxine TC total cholesterol ; TG: Triglycerides; \\ HDL: High-density lipoprotein-cholesterol; LDL-C: Low-density lipoprotein- \\ cholesterol; ROC: Receiver Operating Characteristics; CNS: Central nervous \\ system
}

\section{Acknowledgements}

The authors involved are indebted to all participants and patients involved for their support and cooperation. Acknowledge funding supported by the Jilin Province Science Development Plan [grant numbers [20190304125YY].

\section{Authors' contributions}

XP \& BW: data curation, formal analysis, Writing- Original draft preparation. HW: data curation. LM: data curation. ZD: SNP analyzed through multiplex PCR. YC: conceptualization and project administration, writing - review, project administration. All authors have read and approved the content, and agree to submit it for consideration for publication in your journal.

\section{Funding}

Funding for this work was provided by the Jilin Province Science Development Plan [20190304125YY]. The research funds were mainly used for the design and detection of genetic polymorphisms, as well as for the publication of papers.

\section{Availability of data and materials}

The datasets used and/or analyzed during the current study are available from the corresponding author on reasonable request.

\section{Ethics approval and consent to participate}

All participants included in the study provided written informed consent form. This study was approved by the local Ethics Committee of the First Hospital of Jilin University, Changchun, China (Permission number: 2018 401) and with the 1964 Helsinki declaration and its later amendments or comparable ethical standards.

\section{Consent for publication}

All authors have read and approved the content, and agree to submit it for consideration for publication in your journal.

\section{Competing interests}

The authors declare that they have no competing interests.

\section{Author details}

'Department of Obstetrics, The First Hospital of Jilin University, Xinmin Street 1, Changchun, Jilin Province 130021, China. ${ }^{2}$ Institute of Genetic Technology, Yinfeng Bilogical Group, No. Three Road No. 1109, Shandong, Ji'nan Hi Tech Development Zone Export Processing Zone, Jinan, Shandong Province 250014, China

Received: 4 September 2020 Accepted: 4 December 2020 Published online: 14 December 2020

\section{References}

1. Griffin M, Heazell AEP, Chappell LC, et al. The ability of late pregnancy maternal tests to predict adverse pregnancy outcomes associated with placental dysfunction (specifically fetal growth restriction and preeclampsia): a protocol for a systematic review and meta-analysis of prognostic accuracy studies. Systematic reviews. 2020;9(1):78. doi:https://doi. org/10.1186/s13643-020-01334-5[published Online First: Epub Date]|.

2. Guan L, Fan P, Liu X, et al. Association study between GSTT1 and GSTM1 polymorphisms and risk of preeclampsia in Chinese population. European journal of obstetrics, gynecology, and reproductive biology
2016;204:31 - 5 doi: https://doi.org/10.1016/j.ejogrb.2016.07. 491 [published Online First: Epub Date]|.

3. Macedo TCC, Montagna E, Trevisan CM, et al. Prevalence of preeclampsia and eclampsia in adolescent pregnancy: A systematic review and metaanalysis of 291,247 adolescents worldwide since 1969. European journal of obstetrics, gynecology, and reproductive biology 2020;248:177 - 86 doi: 10 . 1016/j.ejogrb.2020.03.043[published Online First: Epub Date]|.

4. Turbeville HR, Sasser JM. Preeclampsia beyond pregnancy:Long-term consequences for mother and child. American journal of physiology Renal physiology 2020 doi: 10.1152/ajprenal.00071.2020[published Online First: Epub Date].

5. Journal of clinical hypertension (Greenwich, Conn) doi: 10.1111/jch. 13161 [published Online First: Epub Date].

6. Ryu H, Kim J, Kim D, et al. Cellular and Molecular Links between Autoimmunity and Lipid Metabolism. Mol Cells. 2019;42(11):747-54. i: 10. 14348/molcells.2019.0196[published Online First: Epub Date]|.

7. Rambaldi MP, Weiner E, Mecacci F, et al. Immunomodulation and preeclampsia. Best practice \& research Clinical obstetrics \& gynaecology 2019;60:87-96 doi: https://doi.org/10.1016/j.bpobgyn.2019.06.005[published Online First: Epub Date]|.

8. Mitchell J, Kelly J, Kvedaraite E, et al. Foxp3(+) Tregs from Langerhans cell histiocytosis lesions co-express CD56 and have a definitively regulatory capacity. Clinical immunology (Orlando Fla) 2020:108418 doi:https://doi.org/ 10.1016/j.clim.2020.108418[published Online First: Epub Date]|.

9. Zhao H, Liao X, Kang Y. Tregs. Where We Are and What Comes Next? Frontiers in immunology 2017;8:1578 doi: https://doi.org/10.3389/fimmu. 2017.01578[published Online First: Epub Date]|.

10. Gavin MA, Rasmussen JP, Fontenot JD, et al. Foxp3-dependent programme of regulatory T-cell differentiation. Nature. 2007;445(7129):771-5. i: 10.1038/ nature05543[published Online First: Epub Date].

11. Fontenot JD, Gavin MA, Rudensky AY. Pillars Article: Foxp3 Programs the Development and Function of CD4 + CD25 + Regulatory T Cells. Nat. Immunol. 2003. 4: 330-336. Journal of immunology (Baltimore, Md: 1950) 2017;198(3):986 - 92.

12. Lokki Al, Heikkinen-Eloranta JK, Laivuori H. The Immunogenetic Conundrum of Preeclampsia. Frontiers in immunology. 2018;9:2630. i: 10.3389/fimmu. 2018.02630[published Online First: Epub Date]|.

13. Zhang Y, Zhang J, Liu H, et al. Meta-analysis of FOXP3 gene rs3761548 and is 2232365 polymorphism and multiple sclerosis susceptibility. Medicine. 2019;98(38):e17224. i: 10.1097/md.0000000000017224[published Online First: Epub Date]|.

14. Wu Z, You Z, Zhang C, et al. Association between functional polymorphisms of Foxp3 gene and the occurrence of unexplained recurrent spontaneous abortion in a Chinese Han population. Clin Dev Immunol. 2012;2012:896458. doi:https://doi.org/10.1155/2012/896458[published Online First: Epub Date]|.

15. Moresco EM, LaVine D, Beutler B. Toll-like receptors. Current biology: CB. 2011;21(13):R488-93. doi:https://doi.org/10.1016/j.cub.2011.05.039[published Online First: Epub Date]|.

16. Hackl D, Loschko J, Sparwasser T, et al. Activation of dendritic cells via TLR7 reduces Foxp3 expression and suppressive function in induced Tregs. Eur J Immunol. 2011;41(5):1334-43. doi:https://doi.org/10.1002/eji. 201041014[published Online First: Epub Date]|.

17. Xiao W, Liu Z, Lin J, et al. Association of Toll-like receptor 7 and 8 gene polymorphisms with Graves' disease in Chinese Cantonese population. Tissue antigens. 2015;85(1):29-34. i: 10.1111/tan.12479[published Online First: Epub Date].

18. Dutta SK, Tripathi A. Association of toll-like receptor polymorphisms with susceptibility to chikungunya virus infection. Virology. 2017;511:207-13. doi: https://doi.org/10.1016/j.virol.2017.08.009[published Online First: Epub Date]|.

19. Li Y, Zhai S, Li M, et al. [Polymorphisms of TLR7 rs3853839 and rs179010 are associated with susceptibility to and severity of hand, foot and mouth disease caused by enterovirus 71 in male children]. Xi bao yu fen zi mian yi xue za zhi = Chinese. journal of cellular molecular immunology. 2017;33(7):953-58.

20. Jafri S, Ormiston ML. Immune regulation of systemic hypertension, pulmonary arterial hypertension, and preeclampsia: shared disease mechanisms and translational opportunities. American journal of physiology Regulatory integrative comparative physiology. 2017;313(6):R693-r705. doi:https://doi.org/ 10.1152/ajpregu.00259.2017[published Online First: Epub Date].

21. Williams PJ, Searle RF, Robson SC, et al. Decidual leucocyte populations in early to late gestation normal human pregnancy. J Reprod Immunol. 2009;82(1):2431 doi. 1 [published Online First: Epub Date]|. (:: 10.1016/j.jri.2009.08.00. 
22. ACOG Practice Bulletin No. 202: Gestational Hypertension and Preeclampsia. Obstetrics and gynecology 2019;133(1):e1-e25 doi: https://doi.org/10.1097/ aog.0000000000003018[published Online First: Epub Date]|.

23. Chobanian AV, Bakris GL, Black HR, et al Seventh report of the Joint National Committee on Prevention, Detection, Evaluation, and Treatment of High Blood Pressure. Hypertension. (Dallas, Tex: 1979) 2003:42(6):1206-52 doi: https://doi.org/10.1161/01.HYP.0000107251.49515.c2[published Online First: Epub Date]|

24. Di Martino D, Masturzo B, Paracchini S, et al. Comparison of two "a priori" risk assessment algorithms for preeclampsia in Italy: a prospective multicenter study. 2019;299(6):1587-96 doi: https://doi.org/10.1007/s00404019-05146-4[published Online First: Epub Date]|.

25. Levron Y, Dviri M, Segol I, et al. The 'immunologic theory' of preeclampsia revisited: a lesson from donor oocyte gestations. American journal of obstetrics and gynecology 2014;211(4):383.e1-5 doi: https://doi.org/10.1016/ j.ajog.2014.03.044[published Online First: Epub Date]|.

26. Hiby SE, Walker JJ, O'Shaughnessy KM, et al. Combinations of maternal KIR and fetal HLA-C genes influence the risk of preeclampsia and reproductive success. The Journal of experimental medicine. 2004;200(8):957-65. doi: https://doi.org/10.1084/jem.20041214[published Online First: Epub Date]|.

27. Cavoretto PI, Farina A, Miglio R, et al. Prospective longitudinal cohort study of uterine arteries Doppler in singleton pregnancies obtained by IVF/ICSI with oocyte donation or natural conception. Human reproduction (Oxford England). 2020. doi:https://doi.org/10.1093/humrep/deaa235[published Online First: Epub Date]|.

28. Mandia L, Cavoretto P. Evaluation of Uterine Artery Doppler and Estrogen Milieu in Oocyte Donation Pregnancies-A Pilot Study. 2020;10(5) doi: https:// doi.org/10.3390/diagnostics10050254[published Online First: Epub Date]|

29. Masturzo B, Di Martino D, Prefumo F, et al. Higher rate of early-onset preeclampsia in pregnancies following oocyte donation according to increasing maternal age. 2019;300(4):861-67 doi: 10.1007/s00404-019-05291w[published Online First: Epub Date]|.

30. Rasanen J, Girsen A, Lu X, et al. Comprehensive maternal serum proteomic profiles of preclinical and clinical preeclampsia. J Proteome Res. 2010;9(8): 4274-81. doi:https://doi.org/10.1021/pr100198m[published Online First: Epub Date]|.

31. Ozkan ZS, Simsek M, Ihan F, et al. Plasma IL-17, IL-35, interferon- $\gamma$, SOCS3 and TGF- $\beta$ levels in pregnant women with preeclampsia, and their relation with severity of disease. The journal of maternal-fetal \& neonatal medicine: the official journal of the European Association of Perinatal Medicine, the Federation of Asia and Oceania Perinatal Societies. the International Society of Perinatal Obstet. 2014;27(15):1513-7. doi:https://doi.org/10.3109/ 14767058.2013.861415[published Online First: Epub Date]|.

32. Georgiev P, Charbonnier LM, Chatila TA. Regulatory T. Cells: the Many Faces of Foxp3. 2019;39(7):623-40 doi: 10.1007/s10875-019-00684-7[published Online First: Epub Date]|.

33. Zidan HE, Abdul-Maksoud RS, Mowafy HE, et al. The association of IL-33 and Foxp3 gene polymorphisms with recurrent pregnancy loss in Egyptian women. Cytokine. 2018;108:115-19. doi:https://doi.org/10.1016/j.cyto.2018. 03.025[published Online First: Epub Date]|.

34. Lyubomirskaya ES, Kamyshnyi AM, Krut YY, et al. SNPs and transcriptiona activity of genes of innate and adaptive immunity at the maternal-fetal interface in woman with preterm labour, associated with preterm premature rupture of membranes. Wiadomosci lekarskie (Warsaw, Poland: 1960) 2020;73(1):25-30.

35. Hosseini Teshnizi S, Ali-Hassanzadeh M, Gharesi-Fard B. Influence of forkhead box protein 3 polymorphisms (rs2232365, rs3761548) with the outcome of pregnancy: A meta-analysis. 2019 doi: https://doi.org/10.1002/ jcp.28328[published Online First: Epub Date]|.

36. Cao W, Wang $X$, Chen T, et al. Maternal lipids, BMI and IL-17/IL-35 imbalance in concurrent gestational diabetes mellitus and preeclampsia. Experimental therapeutic medicine. 2018;16(1):427-35. doi:https://doi.org/10. 3892/etm.2018.6144[published Online First: Epub Date]|.

37. Barrett HL, Dekker Nitert M, McIntyre HD, et al. Normalizing metabolism in diabetic pregnancy: is it time to target lipids? Diabetes Care. 2014;37(5): 1484-93. i: 10.2337/dc13-1934[published Online First: Epub Date]|. ; ): do.

38. Kingwell BA, Chapman MJ. Future of high-density lipoprotein infusion therapies: potential for clinical management of vascular disease. Circulation. 2013;128(10):1112-21. doi:https://doi.org/10.1161/circulationaha.113. 002683[published Online First: Epub Date]|.
39. Gholami M, Mirfakhraie R, Pirjani R, et al. Association study of FOXP3 gene and the risk of 0020 pre-eclampsia. Clinical and experimental hypertension (New York, NY: 1993) 2018;40(7):613 - 16 doi: 10.1080/10641963.2017. 1411500[published Online First: Epub Date].

40. Norouzian M, Rahimzadeh M, Rajaee M, et al. FoxP3 gene promoter polymorphism affects susceptibility to preeclampsia. Hum Immunol. 2016: 77(12):1232-38. doi:https://doi.org/10.1016/j.humimm.2016.09.001[published Online First: Epub Date]|.

41. Cekin N, Pinarbasi E, Esra Bildirici A. The role of two common FOXP3 gene promoter polymorphisms in preeclampsia in a Turkish population: a casecontrol study. 2020:40(4):495-99 doi: 10.1080/01443615.2019. 1634017[published Online First: Epub Date]|

42. Cekin N, Pinarbasi E. FOXP3 rs3761548 polymorphism is associated with knee osteoarthritis in a Turkish population. 2018;21(10):1779-86 doi: https:// doi.org/10.1111/1756-185x.13337[published Online First: Epub Date]|.

43. Fodor E, Garaczi E, Polyánka H, et al. The rs3761548 polymorphism of FOXP3 is a protective genetic factor against allergic rhinitis in the Hungarian female population. Hum Immunol. 2011;72(10):926-9. doi:https://doi.org/10. 1016/j.humimm.2011.06.011[published Online First: Epub Date]|.

44. Fathima N, Narne P. Association and gene-gene interaction analyses for polymorphic variants in CTLA-4 and FOXP3 genes: role in susceptibility to autoimmune thyroid disease. 2019;64(3):591-604 doi: https://doi.org/10. 1007/s12020-019-01859-3[published Online First: Epub Date]|.

45. Wang T, Marken J, Chen J, et al. High TLR7 Expression Drives the Expansion of CD19(+)CD24(hi)CD38(hi) Transitional B Cells and Autoantibody Production in SLE Patients. Frontiers in immunology. 2019;10:1243. doi:https://doi.org/10.3389/fimmu.2019.01243[published Online First: Epub Date]|

46. Yue M, Feng $L$, Tang SD, et al. Sex-specific association between X-linked Toll-like receptor 7 with the outcomes of hepatitis C virus infection. Gene. 2014;548(2):244-50. doi:https://doi.org/10.1016/j.gene.2014.07.040[published Online First: Epub Date]|.

47. Leite FRM, Enevold C, Bendtzen K, et al. Pattern recognition receptor polymorphisms in early periodontitis. Journal of periodontology. 2019;90(6): 647-54. i: 10.1002/jper.18-0547[published Online First: Epub Date]|.

48. Zhang Y, Suo X, Zhang Y. Association of IL-13, S100B, and TLR-7 Gene Polymorphisms with Enterovirus 71 Infection in Hand, Foot, and Mouth Disease in China. Genetic testing molecular biomarkers. 2019;23(3):18896. doi:https://doi.org/10.1089/gtmb.2018.0239[published Online First: Epub Date]|.

49. Jin WY, Lin SL, Hou RL, et al. Associations between maternal lipid profile and pregnancy complications and perinatal outcomes: a population-based study from China. BMC Pregnancy Childbirth. 2016;16:60. doi:https://doi.org/ 10.1186/s12884-016-0852-9[published Online First: Epub Date]|.

50. Lekva T, Bollerslev J, Norwitz ER, et al. Aortic Stiffness and Cardiovascular Risk in Women with Previous Gestational Diabetes Mellitus. PloS one. 2015; 10(8):e0136892. doi:https://doi.org/10.1371/journal.pone.0136892[published Online First: Epub Date]|.

51. Alemu A, Terefe B, Abebe $M$, et al. Thyroid hormone dysfunction during pregnancy: A review. International journal of reproductive biomedicine (Yazd Iran). 2016;14(11):677-86.

52. Robillard PY, Dekker G, Scioscia M, et al. Increased BMI has a linear association with late-onset preeclampsia: A population-based study. PloS one. 2019;14(10):e0223888. doi:https://doi.org/10.1371/journal.pone. 0223888[published Online First: Epub Date]|.

53. Elhaj ET, Adam I, Alim A, et al. Thyroid Function/Antibodies in Sudanese Patients with Preeclampsia. Front Endocrinol. 2015;6:87. doi:https://doi.org/ 10.3389/fendo.2015.00087[published Online First: Epub Date]|.

54. Khadem N, Ayatollahi H, Vahid Roodsari F, et al. Comparison of serum levels of Tri-iodothyronine (T3), Thyroxine (T4), and Thyroid-Stimulating Hormone (TSH) in preeclampsia and normal pregnancy. Iranian journal of reproductive medicine. 2012;10(1):47-52.

55. Kharb S, Sardana D, Nanda S. Correlation of thyroid functions with severity and outcome of pregnancy. Annals of medical health sciences research. 2013;3(1):43-6. i: 10.4103/2141-9248.109478[published Online First: Epub Date]|

\section{Publisher's Note}

Springer Nature remains neutral with regard to jurisdictional claims in published maps and institutional affiliations. 